Ana Carolina Japur de Sá Rosa-e-SIILVA ${ }^{1}$ Mariani MENDES MAdisSON² Marcos Felipe Silva de-Sá ${ }^{1}$

Rosana Maria ReIs ${ }^{1}$ JULIO CeSAR Rosa-E-SILVA ${ }^{1}$ LuCIA Alves Silva LaRA ${ }^{1}$

Artigo Original

\title{
Macroprolactinemia e hiperprolactinemia intermediária: manifestações clínicas e achados radiológicos
}

\author{
Macroprolactinemia and intermediate byperprolactinemia: clinical \\ manifestations and image
}

Resumo

OBJETIVOS: Caracterizar as pacientes com valores indeterminados de hiperprolactinemia (teste de PEG para identificação de macroprolactinemias com recuperação entre 30 e 65\%) (PRLi) ou macroprolactinemia (PRLm), quanto às características clínicas, como intensidade e variação dos sintomas e presença ou não de tumores no sistema nervoso central. MÉTODOS: Estudo transversal, retrospectivo, de levantamento de prontuários, no qual foram incluídas 24 pacientes com diagnóstico de hiperprolactinemia (PRL>25 ng/dL), em idade reprodutiva, em seguimento em ambulatório de ginecologia endócrina. Foram incluídas 11 pacientes com PRLm e 13 com PRLi. Dos dois grupos (PRLm e PRLi), foram considerados para a análise registros dos dados relativos à idade, à paridade, ao índice de massa corporal, à presença de galactorreia, à infertilidade e ao tumor do sistema nervoso central. Os dados antropométricos foram expressos em média e desvio padrão e, para a comparação entre os grupos quanto à presença de tumor no sistema nervoso central, galactorreia e infertilidade, utilizou-se o teste $t$ de Student. RESULTADOS: A galactorreia foi mais prevalente nas pacientes com PRLi ( $p=0,01)$. Setenta por cento das mulheres com PRLi apresentaram microprolactinoma de hipófise, enquanto que este achado foi evidente em 17\% das mulheres com PRLm ( $p=0,04)$. Dentre as pacientes com PRLm e PRLi, nove não foram investigadas com imagem do sistema nervoso central por apresentarem níveis pouco elevados de prolactina (cinco portadoras de PRLm e quatro de PRLi). Não houve diferença significativa quanto à ocorrência de infertilidade ou de ciclos menstruais irregulares. CONCLUSÕES: Mulheres com hiperprolactinemia intermediária apresentam mais sintomas de galactorreia e maior incidência de tumores do sistema nervoso central do que aquelas com macroprolactinemia.

Abstract

PURPOSE: To characterize patients with indeterminate values of hyperprolactinemia (PEG test for the identification of macroprolactinemias with recovery between 30 and 65\%) (PRLi) or macroprolactinemia (PRLm), in relation to clinical characteristics, such as the presence or absence of symptoms, as well as their intensity and variation, and the presence or absence of central nervous system tumors. METHODS: This is a cross-sectional retrospective survey of records of 24 patients with hyperprolactinemia, in reproductive ages, with prolactin $>25 \mathrm{ng} / \mathrm{dL}$. Eleven women with PRLm and 13 with PRLi were included. Records from the two groups were extracted for analysis: age, parity, body mass index, presence of galactorrhea, infertility, and central nervous system tumor. Anthropometrics data were expressed as mean and standard deviation. To compare groups regarding the presence of central nervous system tumor, galactorrhea, as well as infertility we used the Student's ttest. RESULTS: Galactorrhea was more prevalent in patients with PRLi ( $p=0.01)$. Seventy percent of women with PRLi presented pituitary tumor (microprolactinoma), whereas this finding was evident in $17 \%$ of the PRLm Group (p=0.04). Among the patients with and PRLm PRLi, nine were not investigated with the image of the central nervous system because they have low levels of prolactin (five carriers and four PRLm PRLi). There were no significant differences regarding the occurrence of infertility or irregular menstrual cycles between groups. DISCUSSION: Women with intermediate hyperprolactinemia present more galactorrhea symptoms as well as central nervous system tumors than women with macroprolactinemia.

Correspondêncio

Ana Carolina Japur de Sá Rosa-e-Silvo Avenida Bandeirantes 3.900 - Monte Alegre

CEP: $14049-900$

Ribeirão Preto (SP), Brasil

Recebido

$23 / 12 / 2011$
Trabalho realizado no Serviço de Ginecologia e Obstetrícia da Faculdade de Medicina de Ribeirão Preto da Universidade de São Paulo - USP - Ribeirão Preto (SP), Brasil.

Departamento de Ginecologia e Obstetrícia da Faculdade de Medicina da Universidade de São Paulo - USP - Ribeirão Preto (SP), Brasil.

${ }^{2}$ Curso Acadêmico de Medicina da Faculdade de Medicina da Universidade de São Paulo - USP - Ribeirão Preto (SP), Brasil.

Conflito de interesses: não há. 


\section{Introdução}

A prolactina é um hormônio proteico produzido pela adeno-hipófise, cuja ação está relacionada ao estímulo do desenvolvimento da mama e à produção do leite, exercendo também um papel importante na função reprodutiva feminina. $\mathrm{O}$ fenômeno de maturação do folículo e dos oócitos, bem como a ação fisiológica do corpo lúteo, são dependentes da prolactina que também é responsável por manter o número adequado de receptores do hormônio luteinizante $(\mathrm{LH})$ e estradiol ${ }^{1-2}$. O valor máximo da prolactina, considerado normal para mulheres adultas na fase folicular, varia de 15 até $30 \mathrm{ng} / \mathrm{mL}$ e alguns autores consideram que um valor igual ou superior ao dobro da faixa superior da normalidade poderia ser considerado como francamente anormal ${ }^{3}$.

A hiperprolactinemia interrompe a pulsatilidade do hormônio liberador das gonadotrofinas e, por consequência, influencia negativamente a liberação do LH e FSH, com prejuízo para a esteroidogênese ${ }^{4}$. Isto resulta em alterações dos ciclos menstruais e infertilidade por anovulação.

$\mathrm{O}$ excesso de prolactina pode resultar de disfunções hipotalâmicas ou de tumores hipofisários. Entretanto, muitas vezes é secundário ao uso de alguns medicamentos e pode ainda ser fisiológico em situações como amamentação e a gestação. Entre outros sintomas, a galactorreia está presente em aproximadamente $50 \%$ das mulheres portadoras de hiperprolactinemia ${ }^{5-6}$.

A prolactina é um hormônio bastante heterogêneo e, do ponto de vista de peso molecular, existem três formas principais em circulação: monômero de $23 \mathrm{kDa}$ (small prolactin), dímero de $45 \mathrm{kDa}$ (big prolactin) e macroprolactina de peso molecular entre 150 e 175 kDa (big-big prolactin $)^{7}$. A hiperprolactinemia, gerada às custas de prolactina de baixo peso molecular (fração small), tem sido denominada pela maioria dos autores de hiperprolactinemia verdadeira ${ }^{8}$.

Em condições normais ou em pacientes com hiperprolactinemia sintomática, predomina-se em circulação a forma monomérica ou livre, que é a forma ativa do hormônio. A macroprolactina é constituída, na maioria dos casos, por uma associação entre uma molécula de prolactina e uma de IgG, o que leva a uma meia-vida mais longa e a uma atividade biológica menor?

Em pacientes com hiperprolactinemia (valores superiores a $25 \mathrm{ng} / \mathrm{dL}$ ), o método mais empregado para a pesquisa da existência de quantidades significativas de macroprolactina é o estudo de recuperação pós-precipitação do soro com polietilenoglicol (PEG). Valores recuperados menores que $30 \%$ são considerados positivos para macroprolactinemia ${ }^{10}$, os maiores que $65 \%$ são considerados como microprolactinemia e entre 30 e $65 \%$ são considerados indeterminados (hiperprolactinemia intermediária) ${ }^{10-12}$.
As causas da presença de amostras com valores intermediários devem-se a fatores como: o predomínio da forma dimérica, que apresenta precipitação não-uniforme; a variação do coeficiente de precipitação do teste; a variação das formas monoméricas e das formas de alto peso molecular, entre outros ${ }^{12}$.

Entre as pacientes com hiperprolactinemia, a frequência de casos com predomínio de macroprolactina é de $23 \%^{8-13}$ ou mais. A importância deste fenômeno se deve ao fato de que estas formas têm menor atividade biológica e estão associadas a casos assintomáticos ou oligossintomáticos ${ }^{14}$, e à presença de estudos de imagem do sistema nervoso central normais. Desta maneira, em especial em pacientes cujo quadro clínico não seja característico, a pesquisa de macroprolactinemia é fundamental ${ }^{15}$.

Apesar da pouca informação referente à hiperprolactinemia às custas da macroprolactina, o quadro clínico frustro secundário à baixa atividade de tais moléculas faz com que seu tratamento seja muitas vezes desnecessário. Segundo o guideline para tratamento de prolactinomas da Pituitary Society ${ }^{4}$, as indicações formais de tratamento das hiperprolactinemias se restringem aos casos de pacientes sintomáticos, a macroprolactinomas ou a microprolactinomas assintomáticos com progressão do crescimento. Sendo assim, casos de hiperprolactinemia não-associados a tumor raramente terão indicação de tratamento, já que, na maioria das vezes, são assintomáticos. Não há muita discussão com relação à conduta diante de casos de macroprolactinemia assintomáticos. No entanto, a conduta para os casos com hiperprolactinemia indeterminada, com sintomas que geram pouco desconforto, ou assintomática é, ainda, sujeita a controvérsias, sendo possível que a paciente opte por tratar, ou não, dependendo do desconforto produzido pela doença. Há lacunas no conhecimento quanto à relevância clínica da doença, seja em relação ao comportamento do tumor ou quanto à evolução do quadro. Dessa maneira, este estudo visa caracterizar as pacientes com valores indeterminados de hiperprolactinemia (recuperação entre 30 e 65\%) (PRLi) ou macroprolactinemia (PRLm), quanto às características clínicas, tais como intensidade e variação dos sintomas e presença, ou não, de tumores no sistema nervoso central.

\section{Métodos}

Trata-se de um estudo transversal, retrospectivo, de levantamento de prontuários, no qual foram incluídas pacientes em seguimento no Ambulatório de Ginecologia Endócrina do Hospital das Clínicas de Ribeirão Preto da Universidade de São Paulo (FMRP-USP). As pacientes apresentavam diagnóstico de hiperprolactinemia, mas eram assintomáticas ou apresentavam sintomas clínicos leves (galactorreia somente à expressão da mama e oligomenorreia). 
Este estudo teve a aprovação do Comitê de Ética em Pesquisa (CEP) do Hospital das Clínicas de Ribeirão Preto.

Foram incluídas mulheres em idade reprodutiva, com níveis de prolactina sérica superiores a $25 \mathrm{ng} / \mathrm{dL}$, submetidas à investigação para macroprolactinemia com o uso do pós-teste de PEG ${ }^{16,17}$. Onze mulheres com diagnóstico de macroprolactinemia e 13 com valores indeterminados de hiperprolactinemia foram incluídas. Foram considerados para a análise os registros dos dados relativos à idade, à paridade, ao índice de massa corporal (IMC), à presença de galactorreia, à infertilidade e à presença de tumor do sistema nervoso central. Nos dois grupos (PRLm e PRLi), as dosagens de PRL foram obtidas pelo método da imunoquimioluminescência, utilizando-se kits comerciais específicos da IMMULITE DPC MED LAB, Los Angeles, USA DPC (Immulite ${ }^{\circledR} 2000$ immunoassay analyzer, Diagnostic Products Corporation, Los Angeles, CA, USA).

Nas pacientes com valores de prolactina sérica superiores a $25 \mathrm{ng} / \mathrm{dL}$, procedeu-se ao tratamento da amostra de soro com solução salina de PEG, a qual não precipita a forma monomérica, mas precipita a macroprolactina possibilitando que esta seja removida. Esta técnica consiste em realizar, inicialmente, a dosagem da prolactina total no soro. A seguir, acrescenta-se o polímero polietilenoglicol à amostra testada. Esta composição é misturada em agitador tipo vórtex durante um minuto e, a seguir, centrifugada por cinco minutos, o que leva à precipitação da macroprolactina. Empregando a mesma técnica para a dosagem inicial da PRL, novamente os níveis são dosados à prolactina no sobrenadante recuperado, que representa a prolactina livre ou sua fração de small ${ }^{12}$.

O cálculo da porcentagem de macroprolactina (PRLm) é dado pela fórmula: PRLm (\%)=(PRLm x 2/PRL total)x100. A recuperação da prolactina menor do que $30 \%$ significa que há o predomínio de formas de alto peso molecular, sendo considerados positivos para macroprolactinemia (PRLm), valores maiores que $65 \%$ significam que a amostra tem predomínio de formas monoméricas (microprolactinemia ou hiperprolactinemia verdadeira) e valores de recuperação entre 30 e $65 \%$ são classificados como indeterminados (PRLi). Foram excluídas as mulheres portadoras de hipotireoidismo, acromegalia, insuficiência renal crônica, hepatopatias, condições estas associadas ao incremento da prolactina sérica. Foram também excluídas as usuárias de derivados de haloperidol, fenotiazinas, risperidona, sulpirida e verapamil, os quais exercem efeito antagonista ou reduzem a reserva de dopamina, a qual inibe a secreção de prolactina ${ }^{18}$. Os resultados foram analisados utilizando-se o programa GraphPad Prism, versão 5.00 (GraphPad Software; Inc-2007). Os dados antropométricos foram expressos em média e desvio padrão e, para a comparação entre os grupos quanto à presença de tumor no sistema nervoso central, galactorreia e infertilidade, utilizou-se o teste $t$ de Student. Valores de $\mathrm{p}<0.05$ foram considerados significantes.

\section{Resultados}

Não houve diferença quanto à idade, ao IMC e à paridade entre pacientes com PRLm e aquelas com PRLi (Tabela 1).

$\mathrm{Na}$ avaliação dos sintomas, houve maior prevalência de galactorreia entre pacientes com hiperprolactinemia intermediária $(\mathrm{p}=0,01)$; mas não houve diferença significante com relação à ocorrência de infertilidade ou de ciclos menstruais irregulares (Figura 1).

Quanto à etiologia, 16,7\% das pacientes com macroprolactinemia eram portadoras de tumor, enquanto que $77,8 \%$ das pacientes do grupo com hiperprolactinemia indeterminada apresentavam tumor hipofisário $(\mathrm{p}=0,04)$, como pode ser observado na Figura 2. Dentre as pacientes com PRLm e PRLi, nove não realizaram exame de imagem do sistema nervoso central por apresentarem níveis pouco elevados de prolactina, sendo cinco pacientes com PRLm e quatro com PRLi.

Tabela 1. Características clínicas, epidemiológicas e antecedentes das mulheres portadoras de hiperprolactinemia

\begin{tabular}{l|ccc}
\hline & PRLm média (DP) & PRLi média (DP) & Valor $\mathrm{P}$ \\
\hline Idade & $29,3(9,8)$ & $31,4(9,0)$ & 0,6 \\
Paridade & $1,1(1,6)$ & $0,8(1,2)$ & 0,7 \\
IMC & $23,4(3,5)$ & $24,2(5,1)$ & 0,7 \\
\hline
\end{tabular}

PRLm: macroprolactinemia; PRLi: indeterminado; DP: desvio padrão; IMC: índice de massa corporal.

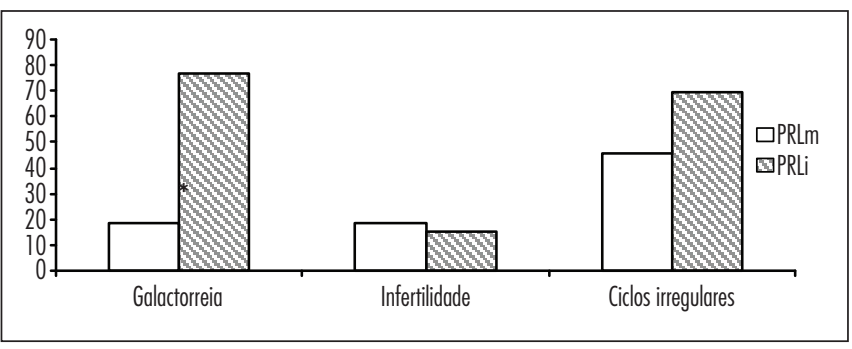

$p=0,01$; PRLi: prolactina indeterminada; PRLm: macroprolactina

Figura 1. Sintomas referidos pelas pacientes.

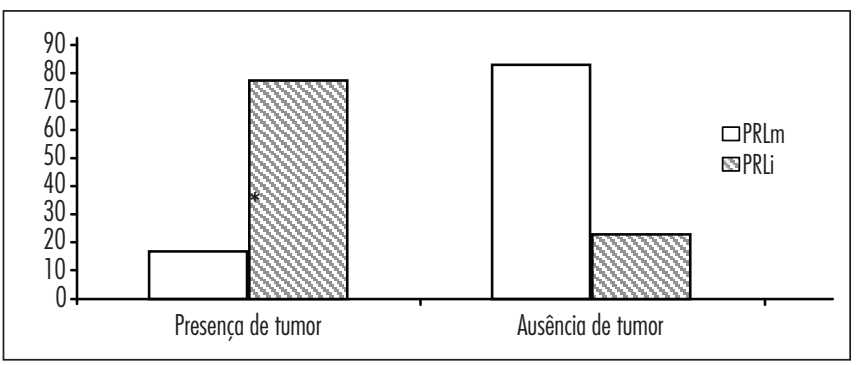

${ }^{*} p=0,04$; PRLi: prolactina indeterminada; PRLm: macroprolactina.

Figura 2. Associação entre presença de imagem no sistema nervoso central e hiperprolactinemia. 


\section{Discussão}

No presente estudo, observou-se maior prevalência de galactorreia entre pacientes com hiperprolactinemia indeterminada quando comparadas àquelas com quadro definido de macroprolactinemia. Há relato de que esse sintoma está presente em $4 \%$ das mulheres portadoras da forma intermediária e em $19 \%$ das mulheres portadoras da forma monomérica ${ }^{8}$. Entretanto, a galactorreia associada a distúrbios menstruais é mais prevalente na forma monomérica ${ }^{19}$. Aproximadamente $40 \%$ das mulheres, com sintomas clínicos de hiperprolactinemia e níveis alterados de prolactina, apresentam nódulos na hipófise ${ }^{20}$.

Em uma porcentagem expressiva dos casos de hiperprolactinemia está presente a forma macro, biologicamente menos ativa, passível de recuperação pós-precipitação do soro com polietilenoglicol. Essa técnica é capaz de caracterizar o tipo de hiperprolactinemia em mais de $85 \%$ dos casos analisados, a um custo muito baixo, evitando-se encaminhamentos desnecessários de pacientes para estudos de imagens ${ }^{11}$. Nos casos restantes, fica o diagnóstico de hiperprolactinemia intermediária e persiste a dúvida sobre suas repercussões e a necessidade, ou não, do tratamento.

Neste estudo, chama a atenção a alta prevalência de tumores no grupo de mulheres com hiperprolactinemia indeterminada $(77,8 \%)$, quando comparadas àquelas portadoras de macropolactinemia (16,7\%). Altas incidências de tumor também foram descritas por Hauache et al. ${ }^{21}, 75 \%$ das pacientes com hiperprolactinemia verdadeira e $11 \%$ das PRLm apresentavam alterações nos exames de imagem do sistema nervoso central. Entretanto, há outros relatos na literatura que mostram associações menos frequentes entre a presença de tumor e a PRLm. Toldy et al..$^{8}$ encontraram que o microadenoma estava presente em $9,8 \%$ das portadoras de macroprolactinemia e em 31,8\% das mulheres com hiperprolactinemia verdadeira. Vilar et al. ${ }^{19}$, em outro estudo, encontraram uma associação de $11 \%$ entre a presença de macroprolactinemia e de microadenoma hipofisário. No entanto, não existem dados na literatura sobre a associação entre hiperprolactinemia indeterminada e a frequência de tumores no sistema nervoso central.

A PRLm responde por um terço ou mais dos casos de hiperprolactinemia ${ }^{8,19,22}$. Já é reconhecido que, nos casos em que há predomínio da macroprolactina, os sintomas são menos comuns (44\%) quando comparados àquelas mulheres portadoras da forma monomérica $(88,5 \%)^{19}$, e nem sempre que estão associados aos níveis da macroprolactina podem refletir a presença de comorbidades ${ }^{22}$. A relação da gravidade dos sintomas clínicos com a prolactina monomérica bem como as diretrizes para o tratamento destes casos já estão bem estabelecidos ${ }^{4}$. Entretanto, ainda faltam evidências na literatura, o significado, o quadro clínico e o manuseio da forma indeterminada da hiperprolactinemia (recuperação $>30 \%$ e $<65 \%$ ), que está presente em 10 a $12 \%$ dos $\operatorname{casos}^{10,11}$.

Em relação à função reprodutiva, o presente estudo não detectou diferença relevante entre os grupos quanto à ocorrência de ciclos menstruais irregulares e relatos de infertilidade. Este achado confirma o de outros autores ${ }^{19}$. Os distúrbios menstruais estão mais associados à forma monomérica ${ }^{19}$, bem como a infertilidade associada à hiperprolactinemia, que está presente em $17 \%$ das mulheres portadoras de macroprolactinemia e em $44 \%$ das portadoras da forma monomérica ${ }^{8}$.

A rotina de screening para macroprolactinemia deveria ser realizada para os casos de pacientes com quadro de hiperprolactinemia, o que, além de prevenir contra a mobilização inadequada de recursos com exames laboratoriais e de imagens $^{23}$, define o tratamento adequado evitando condutas desnecessárias. Ademais, os achados do presente estudo estão em conformidade com aqueles encontrados por outros autores $^{24}$, os quais também sugerem que, em mulheres com macroprolactinemia, os estudos de imagem deveriam ser realizados somente se houver sintomas clínicos associados.

Devido ao fato de que pacientes com hiperprolactinemia intermediária apresentam-se mais sintomáticas, especificamente com relação à presença de galactorreia, e com maior incidência de tumores de hipófise do que pacientes com macroprolactinemia, acredita-se que seja mais prudente que a condução terapêutica deste casos siga os preceitos clínicos semelhantes aos das pacientes com hiperprolactinemia verdadeira, inclusive no que diz respeito à investigação dos tumores do sistema nervoso central. Porém, devido à limitação deste estudo pelo número reduzido de casos, são necessárias mais pesquisas com casuísticas maiores para comprovar esses achados, os quais poderão nortear a elaboração de protocolos específicos para estas pacientes.

No presente estudo, evidenciou-se que a hiperprolactinemia intermediária está associada a uma maior incidência de galactorreia e de tumor no sistema nervoso central.

\section{Referências}

1. Leroy-Martin B, Bouhdiba M, Peyrat JP, Saint PP. Peripheral effects of prolactin in reproductive function. I. Male reproductive function. J Gynecol Obstet Biol Reprod (Paris). 1989;18(1):39-45.

2. Nawroth F. Hyperprolactinaemia and the regular menstrual cycle in asymptomatic women: should it be treated during therapy for infertility? Reprod Biomed Online. 2005; 11 (5):58 1-8.
3. Vieira JGH. Macroprolactinemia. Arq Bras Endocrinol Metab. 2002;46(1):45-50.

4. Casanueva FF, Molitch ME, Schlechte JA, Abs R, Bonert V, Bronstein MD, et al. Guidelines of the Pituitary Society for the diagnosis and management of prolactinomas. Clin Endocrinol (Oxf). 2006;65(2):265-73. 
5. Can M, Guven B, Atmaca H, Acikgoz S, Mungan G. Clinical characterization of patients with macroprolactinemia and monomeric hyperprolactinemia. Kaohsiung J Med Sci. 2011 ;27(5):173-6.

6. Eftekhari N, Mirzaei F, Karimi M. The prevalence of hyperprolactinemia and galactorrhea in patients with abnormal uterine bleeding. Gynecol Endocrinol. 2008;24(5):289-91.

7. Suh HK, Frantz AG. Size heterogeneity of human prolactin in plasma and pituitary extracts. J Clin Endocrinol Metab. 1974;39(5):928 35.

8. Toldy E, Locsei Z, Szabolcs I, Kneffel P, Goth M, Szoke D, et al. Macroprolactinemia in the differential diagnosis of hyperprolactinemia. Orv Hetil. 2003; 144(43):2121-7.

9. Hattori N, Inagaki C. Anti-prolactin (PRL) autoantibodies cause asymptomatic hyperprolactinemia: bioassay and clearance studies of PRL-immunoglobulin G complex. J Clin Endocrinol Metab. 1997;82(9):3107-10.

10. Olukoga AO, Kane JW. Macroprolactinaemia: validation and application of the polyethylene glycol precipitation test and clinical characterization of the condition. Clin Endocrinol (Oxf). 1999;51(1):119-26.

11. Colares AML, Silva NP. Macroprolactina, um método de diferenciação de prolactinas de elevado peso molecular pela precipitação por Polietilenoglicol. Rev Bras Anal Clin. 2006;38(2):75-7.

12. Vieira JG, Tachibana TT, Obara LH, Maciel RM. Extensive experience and validation of polyethylene glycol precipitation as a screening method for macroprolactinemia. Clin Chem. 1998;44/8 Pt 1):1758-9.

13. Dzeranova LK, Goncharov NP, Dobracheva AD, Kolesnikova GS, Giniiatullina EN, Mel'nichenko GA. The diagnostic value of macroprolactin in hyperprolactinemia. Klin Lab Diagn. 2007(10): 16-9.

14. Isik S, Berker D, Tutuncu YA, Ozuguz U, Gokay F, Erden G, et al. Clinical and radiological findings in macroprolactinemia. Endocrine. 2011. [Epub ahead of print].

15. Bagdatoglu C, Bagdatoglu OT, Muslu N, Koseoglu A, Dagtekin $A$, Polat $G$, et al. The importance of macroprolactinemia in the differential diagnosis of hyperprolactinemic patients. Turk Neurosurg. 2008; 18(3):223-7.

16. Smith TP, Suliman AM, Fahie-Wilson MN, McKenna TJ. Gross variability in the detection of prolactin in sera containing big big prolactin (macroprolactin) by commercial immunoassays. J Clin Endocrinol Metab. 2002;87(12):5410-5.

17. Fahie-Wilson MN. Polyethylene glycol precipitation as a screening method for macroprolactinemia. Clin Chem. 1999;45(3):436-7.

18. Shimazu S, Shimatsu A, Yamada S, Inoshita N, Nagamura Y, Usui $T$, et al. Resistance to dopamine agonists in prolactinoma is correlated with reduction of dopamine D2 receptor long isoform mRNA levels. Eur J Endocrinol. 2011. [Epub ahead of print]

19. Vilar L, Naves LA, Freitas MC, Lima M, Canadas V, Albuquerque $\mathrm{JL}$, et al. Clinical and laboratory features greatly overlap in patients with macroprolactinemia or monomeric hyperprolactinemia. Minerva Endocrinol. 2007;32(2):79-86.

20. Cano Sánchez A, Benito López P, Martínez Paredes M, Cara Garcia M, Ribes Bautista R. Pituitary magnetic resonance in women with a clinical suspicion of microprolactinoma. Rev Clin Esp. 1999;199(10):626-31.

21. Hauache OM, Rocha AJ, Maia AC Jr, Maciel RM, Vieira JG. Screening for macroprolactinaemia and pituitary imaging studies. Clin Endocrinol (Oxf). 2002;57(3):327-31.

22. Leslie $\mathrm{H}$, Courtney $\mathrm{CH}$, Bell PM, Hadden DR, McCance DR, Ellis PK, et al. Laboratory and clinical experience in 55 patients with macroprolactinemia identified by a simple polyethylene glycol precipitation method. J Clin Endocrinol Metab. 2001;86(6):2743-6.

23. Strachan MW, Teoh WL, Don-Wauchope AC, Seth J, Stoddart $M$, Beckett GJ. Clinical and radiological features of patients with macroprolactinaemia. Clin Endocrinol (Oxf). 2003;59(3):339-46.

24. de Soárez PC, Souza SC, Vieira JG, Ferraz MB. The effect of identifying macroprolactinemia on health-care utilization and costs in patients with elevated serum prolactin levels. Value Health. 2009; 12(6):930-4. 